\title{
Changing patterns in the workload of a district HIV/AIDS counselling unit 1987-90
}

\author{
R Bor, J Elford, L Campbell, H Salt, R Miller, D Murray, M Johnson
}

\begin{abstract}
Objectives-To describe the changing workload of an HIV/AIDS counselling unit between 1987 and 1990.

Design-Retrospective examination of data collected by the HIV/AIDS counselling unit between 1987-90 on the number of counselling sessions with patients, family members and staff.
\end{abstract}

Setting-An HIV/AIDS counselling unit established in 1987 in a London teaching hospital.

Main outcome measures-Number of new referrals to the HIV/AIDS counselling unit and the number of follow-up sessions. Number of counselling sessions with family members, hospital staff and people making telephone contact with the unit.

Results-New referrals to the HIV/AIDS counselling unit increased from 117 (1987-88) to 926 (1989-90). Follow-up appointments increased from 403 to 2016 in the same period. Telephone counselling sessions increased five-fold, and counselling sessions with family members nearly ten-fold over the three year period. Staff consultations doubled.

Conclusion-The increase in the HIV/AIDS counselling unit's workload may be partly attributable to the rising incidence of AIDS in the community, reflecting earlier patterns of HIV infection. In addition, new HIV/AIDS services were developed in the hospital between 1987 and 1990 . These included the establishment of a same-day HIV test and result clinic; integrated management of patients with HIV/AIDS, with an emphasis on early intervention in HIV infection; specialist services for

The District HIV/AIDS Counselling Unit, Royal Free Hospital

R Bor, L Campbell, H Salt, R Miller, D Murray

Department of Public Health and Primary Care, Royal Free Hospital School of Medicine

$\mathrm{J}$ Elford

Royal Free Hospital, London

M Johnson families, antenatal clinic attenders and others affected by HIV; and the appointment of a designated HIV/AIDS consultant. New approaches to counselling and training health care providers in counselling skills will assume increasing importance in meeting future demand for HIV/AIDS counselling.

\section{Introduction}

HIV infection is not only a medical problem, but also has far-reaching psychosocial implications. ${ }^{1}$ In recognition of this, an HIV/AIDS counselling service was established in Hampstead, London, in 1987. We have already reported on the first two years of this AIDS counselling service. ${ }^{23}$ In this paper we describe the workload during the third year of service and reflect upon some of the changes that have occurred since 1987.

WORKLOAD

New referrals The number of new referrals to the unit has continued to grow. In the year 1987-1988, 117 new referrals were seen in the unit, of whom 41 (35\%) had HIV/AIDS. By 1989-1990 the number of new referrals had increased eight-fold to 926 of whom $123(13 \%)$ had HIV/AIDS (table 1). The three-month moving average of total new referrals to the unit increased from 5 in May 1987 to 124 in February 1990 (fig); the corresponding three-month moving average for new patients with HIV/AIDS rose from 2 to 13 . As in previous years, the majority of new referrals in 1989-90 were out-patients.

Follow-up appointments A significant portion of the workload of the counsellors is taken up with followup appointments and ongoing counselling sessions with existing patients. The number of follow-up sessions with patients has increased nearly five-fold from 403 (1987-88) to 2016 (1989-90) (table 1). Although it was not possible to estimate the number of follow-up sessions per in- or out-patient, our clinical experience indicates that in-patients need more counselling sessions than out-patients. Between 1987 and 1989 we provided, on average, 3.4 follow-up appointments for each new referral. In 1989-90 this ratio decreased to $2 \cdot 2$ follow-up appointments per new referral. 
Table 1 Workload of the Hampstead DHA HIV/AIDS Counselling Unit 1987-90

\begin{tabular}{lclc}
\hline & $1987-88$ & $1988-89$ & $1989-90$ \\
\hline $\begin{array}{l}\text { NEW REFERRALS } \\
\quad \text { In-patients }\end{array}$ & 12 & 40 & 46 \\
$\begin{array}{l}\text { Out-patients } \\
\text { Total (No. HIV/AIDS) }\end{array}$ & $117(41)$ & $176(66)$ & $926(123)$ \\
$\begin{array}{l}\text { FOLLOW-UP } \\
\text { APPOINTMENTS }\end{array}$ & & & \\
$\quad$ In-patients & & & \\
$\quad$ Out-patients & 403 & 334 & 734 \\
$\quad$ Total & 403 & 256 & 1282 \\
TOTAL PATIENT & 520 & 766 & 2016 \\
$\begin{array}{l}\text { CONTACTS } \\
\text { (new referrals + follow-up) }\end{array}$ & & & \\
\hline
\end{tabular}

Total workload Overall, there has been a dramatic increase in the total clinical workload of the counselling unit over the last three years. Five hundred and twenty clinical sessions (both new and follow-up) were recorded in the first year, 766 in the second and 2942 in the third (table 1). In addition, an increasing number of sessions were held with the family, lovers and friends of the index patient; there was nearly a six-fold increase in the number of telephone coun-
Table 2 Hampstead DHA HIV/AIDS Counselling Unit 1987-90: number of contacts with patients' family members, telephone enquiries and staff consultations

\begin{tabular}{lccc}
\hline & $1987-88$ & $1988-89$ & $1989-90$ \\
\hline Family members & 24 & 104 & 230 \\
Telephone counselling & 341 & 543 & 1818 \\
Staff consultations & 666 & 653 & 1354 \\
\hline
\end{tabular}

selling sessions and requests for HIV antibody test appointments; and consultations with colleagues over the psychosocial management of patients also increased (table 2).

Teaching, research and administrative duties comprise additional activities of the AIDS counsellors.

\section{Discussion}

Statistics from the CDSC reveal a growing number of people with HIV infection and AIDS nationwide. ${ }^{4}$ This is inevitably reflected in the number of people who attend medical centres throughout the country. The trend in this central London teaching hospital HIV/AIDS counselling unit (which does not include statistics from our Regional Haemophilia Centre) has

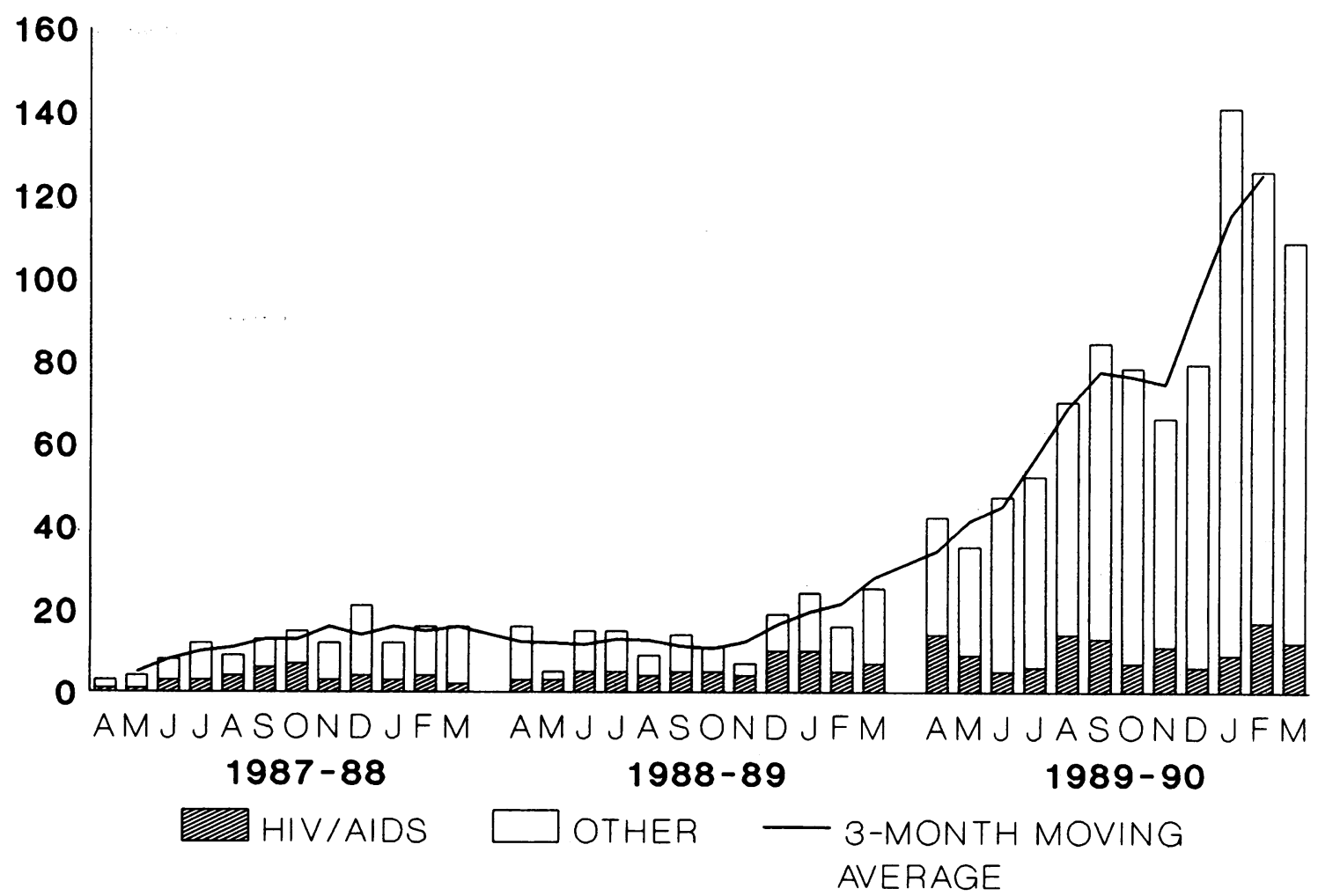

Figure Number of new referrals to the HIV|AIDS Counselling Unit by month April 1987-March 1990. 
has been a sustained increase in both new patients attending the service as well as follow-up appointments for existing patients. The increasing workload may be attributable to a number of factors and the main ones are listed below:

\section{Comprehensive HIV|AIDS care}

New developments in HIV/AIDS care at the Royal Free Hospital have undoubtedly attracted patients to the hospital and to the HIV/AIDS counselling unit from within, as well as outside the district. A fulltime consultant physician in HIV/AIDS was appointed in January, 1989, the first appointment of its kind in the NHS. The consultant sees all patients, both asymptomatic and symptomatic, who attend either for HIV testing or follow-up care. Continuity of medical care has proved popular with patients. A designated in-patient unit on a general medical ward has also attracted patients to the hospital. They simultaneously benefit from co-ordinated in-patient care from specialist HIV/AIDS doctors, nurses and counsellors but do not face the potential stigma and loss of confidentiality associated with being on an AIDS ward. The appointment of a community nurse specialist, the development of a unit for drug users and the involvement of our local Citizens' Advice Bureau have further extended our range of services. In addition, some patients diagnosed in other AIDS units who live in our catchment area have transferred their care to the Royal Free Hospital.

\section{Change in emphasis in treatment}

A shifting emphasis in following up and treating asymptomatic HIV infected patients, and not only those with an AIDS diagnosis, has concomitantly led to an increasing number of patients seeking an HIV test and attending an HIV clinic. Counsellors and their medical colleagues are likely to see their patients over a longer period of time, although the sessions may range in frequency from once a week to once every third month, on an out-patient basis. Publicity concerning the advantages and benefits of early diagnosis and treatment may partially account for the increasing number of new patients seeking counselling and HIV antibody testing. In addition, treatment of patients with HIV and AIDS is shifting from a model of acute care to chronic disease management. As a consequence, patient contact extends over a longer period of time than in the past. Living with uncertainty and deteriorating health over an extended period of time leads to an increased involvement by professional counsellors.

Same-day HIV antibody test clinic A same-day HIV antibody test-and-result clinic was set up in March 1989. It has proved popular due to its convenience and the speed with which the result becomes available. The organisation of the service and the workload in its first year has been described in a separate paper. ${ }^{5} \mathrm{~A}$ large number of the new referrals to the counselling service, as well as telephone counselling sessions in 1989-90, can be attributed to this new service. Each patient is seen at least once for a pre-test counselling session (new referral) and again at the time the result is given (follow-up). Most people who come to the Same-day HIV test clinic only require one follow-up appointment, since their result is negative. This partly explains the decline in the ratio of follow-up appointments to new referrals seen in the last year.

Those who are found to be HIV antibody positive are then offered continuing care by doctors and counsellors. Some HIV antibody negative patients are also seen for additional follow-up sessions as their presentation to the counselling service may be indicative of a "worried-well" problem. ${ }^{6}$ A few patients request counselling to help them deal with habitual HIV risk-taking behaviour and other personal and relationship difficulties.

\section{Specialist counselling services}

All of the counsellors working in the service are trained in family counselling and family interviewing skills. This dimension of the counselling service has expanded as more patients seek advice and support for their families and close contacts. Relationship problems that stem directly or indirectly from the diagnosis can be addressed within the counselling setting. Ideally, effective family and partner support should reduce the need for professional counselling, and for this reason family counselling is given high priority in the specialist counselling services on offer to patients. Apart from the service for families and partners of patients, the counselling unit provides a range of other specialist HIV/AIDS counselling services. Mothers attending the antenatal clinic who would like to be tested for HIV are seen by a counsellor who is present at that clinic for one session per week. Patients attending the HIV/AIDS unit are also seen for stress management, housing and benefits advice and discussion about entering into drugs trials. As the prognosis for patients under treatment improves so the demand on these services increases. "Worried-well" patients are also seen by counsellors, who work closely with liaison psychiatrists and psychologists.

\section{Growth in the HIV/AIDS counselling team}

The number of full-time professional counsellors in the counselling unit has increased each year from one in 1987-8, to two in 1988-9 and three in 1990. The growth in the number of counsellors increases the potential for counselling sessions in the unit. In the health service, where human and other resources are limited, we recognize that the counselling team cannot expand indefinitely. For this reason we are 
developing "brief counselling" approaches" and teaching other hospital-based professionals basic HIV/AIDS counselling techniques ${ }^{8}$ in order to meet the increasing demand for counselling services and to spread skills and expertise.

\section{Conclusion}

As anticipated in our first year, there has been a considerable increase in the number of new referrals and follow-up counselling sessions over the three year since the unit opened. A change in emphasis in treating HIV infection and not only AIDS has probably led to an increase in the number of people seeking HIV tests and attending for follow-up care. As HIV infection becomes more a chronic rather than acute medical problem, so the organisation and provision of medical and support services will need to be adapted accordingly. This raises the possibility of direct access specialist units in hospitals and shared care with general practitioners in the community and physicians in district general hospitals, similar to the model of services now provided in haemophilia and diabetes care. Projections for the rate of increase of HIV positive cases and AIDS patients in the UK were revised downwards for the 1990 s. $^{9}$ Nonetheless, the number of patients with HIV/AIDS will continue to increase, reflecting patterns of infection some ten years ago. Since resources for counselling are limited, brief counselling approaches will need to be developed. Training courses in HIV/AIDS counsell- ing for a wide range of health care staff will need to be provided if the increasing demand on HIV/AIDS services is to be met. Such training may also allow a wider range of health care workers to integrate issues surrounding HIV/AIDS into their daily professional practice.

Address for correspondence: Dr Robert Bor, AIDS Counselling Unit, Royal Free Hospital, Pond Street, London NW3 2QG, UK

1 Green J. Counselling for HIV infection and AIDS: the past and the future. AIDS Care 1989;1:5-10.

2 Bor R, Elford J, Miller R, et al. Workload of a new district AIDS counselling unit, April 1987 to March 1988. Genitourin Med 1989;65:113-6.

3 Bor R, Elford J, Perry L, et al. New initiatives in a district AIDS counselling unit, 1988-89. Int J STD AIDS 1990;1:110-3.

4 CDSC Communicable Disease Report 90/44 PHLS 1990: London.

5 Squire B, Elford J, Bor R, et al. An open access clinic providing HIV-1 antibody test results on the same day: the first twelve months. BMJ 1991 (in press).

6 Miller D, Acton T, Hedge B. The worried well: their identification and management. $J$ Roy Soc Med 1988;22:158-65.

7 Miller R, Bor R. AIDS: A Guide to Clinical Counselling London: Science Press.

8 Bor R, Elford J, Salt $H$, et al. Training for HIV/AIDS counselling in a clinical setting. Health Hygiene 1991;12: 16-20.

9 Day N. AIDS in England and Wales to end 1993: projections using data to end September 1989. Communicable Disease Report 1990;PHLS:Supplement.

Accepted for publication 1 March 1991 